\title{
Clinical Outcomes of Cranioplasty Using a Customized Artificial Bone Flap Made by a 3D Printing Technique
}

\author{
Jin Ho Lee, Yong Woo Shim, Sung-Tae Kim, Won Hee Lee, Keun Soo Lee, Sung Hwa Paeng, Se Young Pyo
}

Department of Neurosurgery, Inje University College of Medicine, Busan Paik Hospital, Busan, Korea

Received: August 25, 2021

Accepted: September 6, 2021

Corresponding Author:

Sung-Tae Kim, MD

Department of Neurosurgery, Inje University College of Medicine, Busan Paik Hospital, 75 Bokji-ro, Busanjin-gu, Busan 47392, Korea Tel: +82-51-890-6144

Fax: +82-51-898-4244

E-mail: kimst015@hanmail.net

\begin{abstract}
Objective
To analyze the outcomes of using a customized artificial skull bone flap made with a three-dimensional (3D) printing technique for cranioplasty and compare them with those of the autobone technique.

Method

Between December 2018 and February 2020, 24 cranioplasties were performed for 24 hemispheres using a customized artificial bone flap made by 3D printing. Simultaneously, 19 cranioplasties for 19 hemispheres were performed using an autobone. Three patients underwent cranioplasty using both a customized artificial bone flap and autobone for each hemisphere. Patient's demographics, reason for craniectomy, interval between craniectomy and cranioplasty, surface area of the skull defect and bone flap, bone flap coverage of the defect, cranioplasty-related factors, and clinical outcome were assessed.

Results

Forty patients who underwent cranioplasty (bone flap, 21; autobone flap, 16; and artificial bone/ autobone flaps, 3) were enrolled. The artificial bone flap covered more skull defects than the autobone flap $(98.6 \%$ vs. $90.9 \%, \mathrm{p}=0.000)$. There were two and six operation-related complications in the artificial bone flap and autobone flap groups, respectively $(\mathrm{P}=0.061)$. The subtemporal area was completely covered in the artificial bone flap group. Two patients had an infection of the autobone flap and had it replaced by a 3D printing flap. No patient showed a reduction in the modified Rankin Scale score after surgery, and the clinical course was confirmed to have improved.

\section{Conclusions}

Cranioplasty using customized artificial bone flap made by $3 \mathrm{D}$ printing technique was effective for covering the skull defect and tends to have a low complication rate compared to the autobone.
\end{abstract}

Keywords: Autobone; Bone flap; Cranioplasty; Craniotomy; Printing; Three-dimensional

\section{INTRODUCTION}

Cranioplasty is required to restore the appearance of the skull, protect intracranial tissue, and improve neurological function ${ }^{1,2)}$. Autologous bone flaps are usually the first choice at most institutions, as they are readily available at no additional cost and yield ex-

Copyright (C) 2021 The Korean Neurointensive Care Society

This is an Open Access article distributed under the terms of the Creative Commons Attribution Non-Commercial License (http://creativecommons.org/licenses/by-nc/4.0/) which permits unrestricted non-commercial use, distribution, and reproduction in any medium, provided the original work is properly cited. 
cellent outcomes ${ }^{3,4)}$.

Although autologous bone remains the first choice for repair, it is not always used because of infection, fragmentation, bone resorption, or other causes ${ }^{5,6)}$, which has led to the use of synthetic alternatives ${ }^{7)}$. The ideal cranioplasty material is nonmagnetic, radiolucent, light, sterilizable, and easily affixed to the skull. Currently, the three most popular nonbiological materials used for this purpose are polymethylmethacrylate (PMMA), hydroxyapatite, and titanium meshes or plates. However, trials using materials such as polyether ether ketone and porous polyethylene are also being conducted $^{8)}$.The procedures for using these materials vary between centers, with some preferring to shape the implant intraoperatively, while others choose to create custom-made plates preoperatively using computed tomography (CT) or stereolithography data ${ }^{8,9,10}$.

Three-dimensional (3D) printing techniques are evolving, and their application in medical technology, such as utilizing it to make artificial bone flaps for various surgeries is increasing, ${ }^{11,12}$. This study aimed to analyze the outcomes of using a customized artificial skull bone flap made by a 3D printing technique for cranioplasty, compared with autobone. Significant results were confirmed as statistical differences between the results of cases.

\section{MATERIALS AND METHODS}

This study was a retrospective study of medical records, including a survey of satisfaction after 3D cranioplasty (3DC). All procedures performed in studies involving human participants were in accordance with the ethical standards of the Ethical Committee of Busan Paik Hospital of Inje Medical University and with the Helsinki Declaration. Since this study was conducted retrospectively, it was exempted from that criterion.

\section{Patient data}

The data of 40 patients who underwent cranioplasty between December 2018 and February 2020 were included in the study. All patients in this study were included in accordance with criteria. No patients were excluded. A total of 24 cranioplasties for 24 hemispheres using a customized artificial bone flap made by $3 \mathrm{D}$ printing technique were performed. Simultaneously, 19 cranioplasties were performed for 19 hemispheres using autobone. Among them, three patients underwent cranioplasty using both customized artificial bone flap and autobone for each hemisphere.

We assessed the patients' demographics, reason for craniectomy, interval between craniectomy and cranioplasty, operation time, bleeding volume, surface area of the skull defect and bone flap, bone flap coverage of the defect, cranioplasty-related factor, comparison between the two groups, and 6-month clinical outcome in- cluding telephone survey of satisfaction after 3DC.

\section{Cranioplasty style and material}

All customized artificial 3D printing bone flaps were made by PRYMARY. (CUSMEDI, South Korea), which is a patient-specific implant using titanium alloy (Ti6Al4V ELI in accordance with ASTM F136\&F3001-14) produced via selective laser melting additive manufacturing/3D printing. Preoperative CT scan data (1 mm slice thickness) were converted to 3D images (Aview Modeler, CORELINE, South Korea), and the implant was designed using a mirror-imaging process of the normal site of the cranium using Materialize Magics (3D Systems, USA). The surface of the 3D implant model was processed using a smoothing technique by the Meshmixer (Ver. 3.5, Autodesk, United States of America).

The operation procedure was as follows. Under general endotracheal anesthesia, the patient was placed on the operating table in the supine position and the head was positioned. The whole scalp was prepared with scrub-povidone, povidone, and draped. A scalp incision was made along the previous surgical scar, and bleeding was controlled with a bovi and bipolar coagulator. The scalp flap was reflected, and the adhesied part of the scalp was adhesiolized with a No. 15 blade and bovi monopolar coagulator. Bleeding from the inner surface of the scalp and muscle was controlled with a bipolar coagulator.

The soft tissue overlying the dura was separated from the dura. The 3D printing flap was fixed with screws, and the autobone flap was fixed with miniplates and screws. After central tagging and meticulous bleeding control, the wound was closed in layers. The patients tolerated the procedure described above. (Fig. 1)

\section{Measurement of craniectomy lesions and defects}

In the case of 3DC, the defect values and flap surface area were measured using Mimics Research Version 19.0 and 3-matic Research 11.0 made by Materialize. (Leuven, Belgium) After obtaining the craniectomy area and autobone flap area using Materialise Mimics and 3-matic, respectively, the subtraced area was defined as the defect area. The average circumference of the craniectomy area and autobone flap was calculated, and the average defect width was calculated using the formula described below.

verageydefectyofycranioplasty $=\frac{\text { Areatoftdefect }}{(\text { Circumstance }) \text { of }) \text { Autobone }) \text { flap }+ \text { Craniectomy }) / 2}$

Fig. 2 illustrates the defects of craniectomy and bone flap and the parameters of the formula.

\section{Statistical analysis}

Statistical analyses were performed using SPSS for Windows 

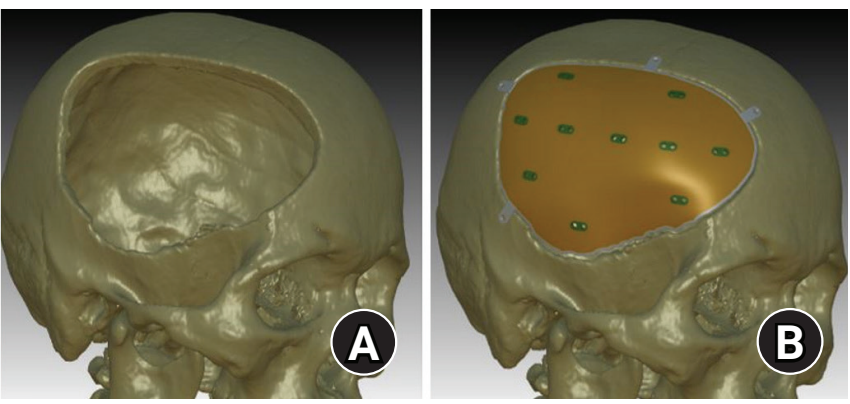

Fig. 1. Illustration of 3D cranioplasty. 3D reconstruction of a skull with a large cranial defect (A) customized artificial 3D printing flap covered on skull defect (B).

3D: Three-dimensional.

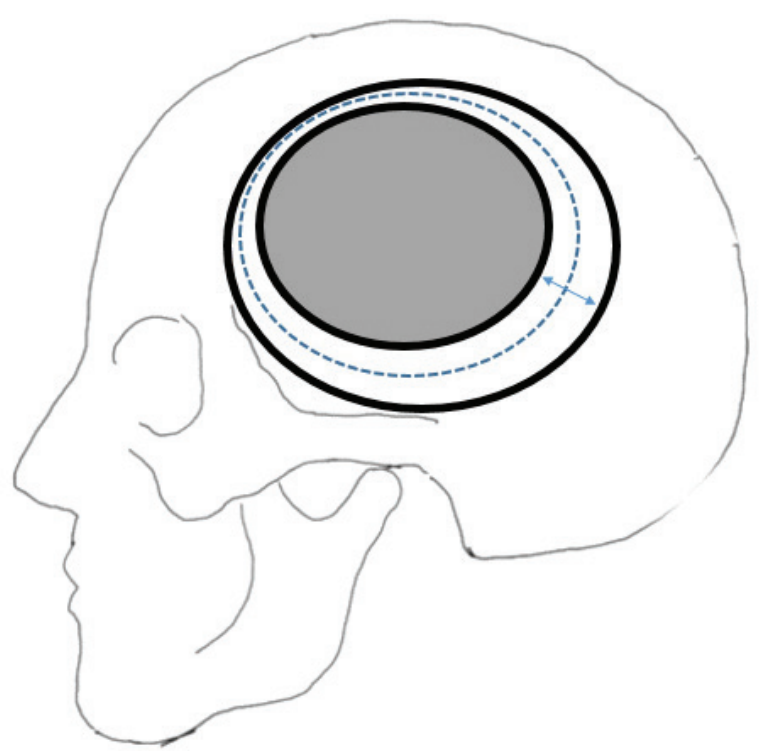

Fig. 2. The formula of a skull defect, bone flap, and parameters of the formula. The external bold-lined oval is a skull defect, and the internal bold-lined oval is a bone flap. The dotted lined oval between two bold-lined ovals is the average length of the circumstance of the bone flap and skull defect. The bidirectional arrow indicates the average of the defect in cranioplasty.

(version 26; SPSS, Chicago, IL, USA). Univariate analysis was performed with the time interval between craniectomy and cranioplasty, hemisphere (right/left/both), operation time, blood loss, complications, circumstance of the autobone flap, circumstance of craniectomy, surface area of the skull defect, surface area of the bone flap, bone-flap coverage of the skull defect, area of defect $\left(\mathrm{mm}^{2}\right)$, and average of defect $(\mathrm{mm})$ as factors using the Student's t-test, chi-square test, or Fisher's exact test due to the expected frequency.

\section{RESULTS}

Forty patients were included in this study (average age, 51.1 years; 21 male). 3DC was performed in 24 patients (average age, 49.5 years; 16 male) and autobone cranioplasty (AC) was performed in 19 patients (average age 49.7 years; 8 male). Among them, 26 patients had cerebrovascular accidents (cerebral infarction, aneurysmal spontaneous subarachnoid hemorrhage, spontaneous intracranial hemorrhage (ICH), one brain abscess, and 13 head traumas. The mean interval between craniectomy and cranioplasty was 5.6 months (3DC group, 6.6 months; AC group, 4.3 months; $\mathrm{p}=0.123$ ). The affected hemispheres were 27 on the right and 17 on the left ( $p=0.096$; in one case, AC for both frontal craniectomies were performed in one step. The average operation time was 167.3 $\mathrm{min}$ (3DC group, $168.5 \mathrm{~min}$; AC group, 165.8 $\min ; \mathrm{p}=0.889$ ). The average blood loss during operations was 172 $\mathrm{mL}$ (3DC group, $184 \mathrm{~mL}$; AC group, $156156 \mathrm{~mL}$; $\mathrm{p}$ = 0456). Cranioplasty-related complications occurred in eight cases (3DC group, two; AC group, six; $\mathrm{p}=0.061$ ). There were no statistically significant differences in age, sex, interval between craniectomy and cranioplasty, operation time, and blood loss between the two groups. In four patients in the AC group, cranioplasty was accompanied by bone cement. (Table 1 )

The average of circumference of craniectomy was $403 \mathrm{~mm}$ (3DC group, $399 \mathrm{~mm}$; AC group, $408 \mathrm{~mm} ; \mathrm{p}=0.730$ ) and surface area of skull defect was $103.93 \mathrm{~cm}^{2}$ (3DC, $106.74 \mathrm{~cm}^{2}$; AC, 100.39 $\left.\mathrm{cm}^{2} ; \mathrm{p}=0.503\right)$. The average bone flap circumference was 363 $\mathrm{mm}$. (3DC, $366 \mathrm{~mm}$; AC, $359 \mathrm{~mm}$; $\mathrm{p}=0.687$ ), and average of surface area of the bone flap was $98.95 \mathrm{~cm}^{2}$ (3DC, $105 \mathrm{~cm}^{2}$, AC, 90 $\left.\mathrm{cm}^{2} ; \mathrm{p}=0.112\right)$. However, bone flap coverage of the skull defect was 95.2\% (3DC, 98.6\%, AC, 90.9\%; $\mathrm{p}=0.000$ ), and the area of defect was $498 \mathrm{~mm}^{2}$ (3DC, $146 \mathrm{~mm}^{2}, \mathrm{AC}, 943 \mathrm{~mm}^{2}$; P-value 0.000 ). In addition, the average size of defect was $1.23 \mathrm{~mm}$ (3DC, $0.35 \mathrm{~mm}, \mathrm{AC}, 2.35 \mathrm{~mm} ; \mathrm{p}=0.000)$. In this data, bone flap coverage, area of defect, and average of defect showed statistically significant differences $(\mathrm{p}<0.000)$, and are summarized in Table 2. Supplementary Fig. 1 shows the high skull defect coverage of the 3DC.

In the case of cranioplasty-related complications, there were two operation-related complications (one case of cosmetic issues and one case of ICH after cranioplasty) in the 3DC group, and six (one episode of extradural hematoma after cranioplasty, one case of cosmetic issue, two cases of bone floating, and two cases of infection requiring bone-flap removal) in the AC group. $(\mathrm{p}=0.061)$ Table 3 summarizes the data. Temporal muscle atrophy was inevitable even with 3DC. (Supplementary Fig. 2) Regarding AC, bone flap floating occurred in two cases. (Fig. 3)

In the 3DC group, the preoperative modified Rankin Scale 
Table 1. Basic characteristics of the 3D cranioplasty and autobone cranioplasty groups*

\begin{tabular}{|c|c|c|c|c|}
\hline & Total & $3 \mathrm{D}$ cranioplasty $(\mathrm{n}=24)$ & Autobone cranioplasty $(\mathrm{n}=19)^{* *}$ & p-value \\
\hline Age (SD) & $51.1(15.07)$ & $49.5(15.75)$ & $49.7(16.40)$ & $0.955^{+}$ \\
\hline Sex Male (Female) & $21(19)$ & $16(8)$ & $8(11)$ & $0.107^{\ddagger}$ \\
\hline \multicolumn{5}{|l|}{ Cause of craniectomy } \\
\hline Head trauma & 13 & 10 & 5 & \\
\hline Cerebral infarction & 4 & 1 & 3 & \\
\hline Aneurysmal SAH & 12 & 6 & 7 & \\
\hline Spontaneous ICH & 10 & 6 & 4 & \\
\hline Brain abscess & 1 & 1 & 0 & \\
\hline Interval between craniectomy and cranioplasty, months (SD) & $5.6(5.39)$ & $6.6(6.85)$ & $4.3(2.18)$ & $0.123^{+}$ \\
\hline Hemisphere(R/L/Both) & & $15 / 9 / 0$ & $11 / 7 / 1^{* * *}$ & $0.096^{\S}$ \\
\hline Operation time, mins (SD) & $167.3(63.3)$ & $168.5(54.4)$ & $165.8(74.7)$ & $0.889^{+}$ \\
\hline Blood loss, mL (SD) & $172(123.4)$ & 184(109.6) & $156(140.3)$ & $0.456^{+}$ \\
\hline Complication cases & 8 & 2 & 6 & $0.061^{\S}$ \\
\hline
\end{tabular}

*Three patients underwent both 3D cranioplasty and autobone cranioplasty in a stepwise manner.

** Three patients underwent both $3 \mathrm{D}$ cranioplasty and autobone cranioplasty in a stepwise manner.

***In one case, autobone cranioplasty for both frontal craniectomy was performed in one step.

${ }^{\dagger}$ Student T-Test.

${ }^{\ddagger}$ Chi-square.

${ }^{s}$ Fisher's exact test.

3D: Three-dimensional, ICH: Intracranial hemorrhage, SAH: Subarachnoid hemorrhage, SD: Standard deviation.

Table 2. Parameters about craniectomy and bone flap

\begin{tabular}{|c|c|c|c|c|}
\hline & Total & $3 \mathrm{D}$ cranioplasty $(\mathrm{n}=24)$ & Autobone cranioplasty $(\mathrm{n}=19)^{*}$ & $\mathrm{p}$-value \\
\hline Circumstance of craniectomy, mm (SD) & $403(81)$ & $399(46)$ & $408(113)$ & 0.73 \\
\hline Surface area of the skull defect, $\mathrm{cm}^{2}$ (SD) & $103.93(30.4)$ & $106.74(25.3)$ & $100.39(36.3)$ & 0.503 \\
\hline Circumstance of bone flap, mm (SD) & $363(61)$ & $366(42)$ & $359(80)$ & 0.687 \\
\hline Surface area of the bone flap, $\mathrm{cm}^{2}(\mathrm{SD})$ & $98.95(29.33)$ & $105.285(25.2)$ & $90.95(32.7)$ & 0.112 \\
\hline Bone flap coverage of the skull defect (\%) & $95.2 \%(7.1)$ & $98.6 \%(1.0)$ & $90.9 \%(1.5)$ & 0.000 \\
\hline Area of defect, $\mathrm{mm}^{2},(\mathrm{SD})$ & $498(756)$ & $146(593)$ & $943(715)$ & 0.000 \\
\hline Average of defect, mm (SD) & $1.23(1.85)$ & $0.35(1.44)$ & $2.35(1.74)$ & 0.000 \\
\hline
\end{tabular}

*In four patients bone cement was used along with autobone 3D: Three-dimensional; SD: Standard deviation.

Table 3. Detailed information on cranioplasty-related complications

\begin{tabular}{lclll}
\hline & Total & 3D cranioplasty $(\mathrm{n}=24)$ & Autobone cranioplasty $(\mathrm{n}=19)$ & $\mathrm{p}$-value \\
\hline Complications & 8 & 2 & 6 & EDH $(1)$ \\
& Cosmetic issue $(1)$ & Cosmetic issue $(1)$ \\
& $\mathrm{ICH}(1)$ & Bone floating $(2)$ \\
& & Infection $(2)$
\end{tabular}

3D: Three-dimensional, EDH: Extradural hematoma, ICH: Intracranial hemorrhage.

(mRS) scores were as follows: (4 points, 6 patients; 5 points, 6 patients). The postoperative scores were as follows: ( 4 points, 7 patients; 5 points, 0 patients). In addition, preoperative $\mathrm{mRS}$ scores of the AC group were as follows: (4 points, 5 patients; 5 points, 1 patient). The postoperative scores were as follows: (4 point, 3 patients; 5 point, 0 patients). There was no patient with a lowering of $\mathrm{mRS}$ after the operation in any group, and the clinical course was confirmed to have improved. (Table 4)

\section{DISCUSSION}

A large format (i.e., $>25 \mathrm{~cm}^{2}$ ) cranioplasty is a challenging procedure not only from a cosmetic standpoint, but also in terms of ensuring that the patient's brain is well protected from direct trau- 


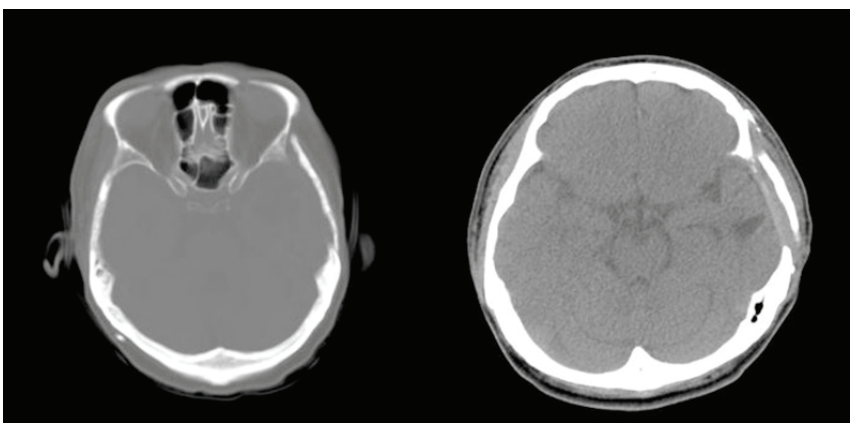

Fig. 3. Temporal bone floating after autobone cranioplasty. This may have occurred because of incomplete temporal muscle dissection.

Table 4. Clinical status in pre and post cranioplasty

\begin{tabular}{lccccc}
\hline \multirow{2}{*}{$\begin{array}{l}\text { Modified } \\
\text { Rankin Scale }\end{array}$} & \multicolumn{2}{c}{3 3D cranioplasty $(\mathrm{n}=24)$} & & \multicolumn{2}{c}{ Autobone cranioplasty $(\mathrm{n}=19)$} \\
\cline { 2 - 3 } \cline { 5 - 6 } & Pre & Post & & Pre & Post \\
\hline 0 & 1 & 6 & & 4 \\
1 & 6 & 2 & & 4 & 3 \\
2 & 3 & 3 & & 4 & 4 \\
3 & 2 & 6 & 4 & 5 \\
4 & 6 & 7 & 5 & 3 \\
5 & 6 & 0 & 1 & 0 \\
\hline
\end{tabular}

3D: Three-dimensional.

ma, sinking flap syndrome, and syndrome of the trephined. Until recently, when a patient's cranial flap was unavailable, it was difficult to achieve these goals. It is now possible to 3-D-print patient-specific implants from a variety of polymer, ceramic, or metal components. A 3D printing design may be used to imitate the previous external shape of the skull that will become well integrated into the skull while providing beneficial distribution of mechanical force in the event of trauma ${ }^{13)}$. Recently, 3D printing flaps have been increasingly used in patients undergoing cranioplasty ${ }^{14,15)}$.

If $3 \mathrm{D}$ printing flaps are used, there would be advantages in the infection ratio and cosmetic area ${ }^{16}$. In this study, we evaluated and compared the defects between the autobone flap and 3D printing flap for cosmetic points. In general, craniectomy is focused on the temporal area to achieve maximal decompression. With the increase in subtemporal decompression, the prognosis of the patient would improve if the shape of the midbrain is restored and the cerebrospinal fluid flow is smooth ${ }^{17)}$. However, the removed skull bone can remain as temporal bone defects that are not well covered in AC. In the case of the 3D printing flaps, the temporal defect can be solved by imitating the unaffected skull.

There are multiple holes in the 3D printing flap. Using these multiple holes, sufficient dural tenting can be performed to reduce additional manipulation. When using an autobone, a suture hole for tenting must be made using a drill, hand manipulation is in- volved, and the number of holes is limited. In a 3D printing flap, dural tenting can be performed more easily because of multiple holes. However, epidural fluid collection occurred in both groups in this study. Such complications vary depending on the surgeon. There was a difference in the dissection of the adhesion site and epidural space control. To prevent epidural fluid collection, it is important to meticulously check the leakage point and perform duroplasty. In the case of autobone complications that were not seen in $3 \mathrm{D}$ printing cranioplasty, calcified deposits were found in the soft tissue. In the case of AC, cement is used as a complementary method for the defect. Forming cement for cranioplasty can increase the infection risk by using hand manipulation and develop complications in which a fragment of cement is found in soft tissue, as in the case of a patient in this study.

Additional treatment caused by a cranioplasty defect may increase the risk of infection due to an increase in operation time and hand manipulation. However, in the case of 3DC, all defects were designed to be covered, and this might have been better than the autobone flap in this regard. Postoperative infection was not observed in the 3DC group. If an infection develops after AC, it is impossible to reuse the autobone because it may provide bacterial culture. Conversely, a 3D printing flap is formed using titanium, which has a lower infection rate. In cases of infection caused by using an autologous skull bone flap, the infection must be overcome through revision surgery using a 3D printed bone flap. In addition, resorption may occur due to apoptosis of the bone flap in the $\mathrm{AC}^{18)}$. In such cases, the defect at the cranioplasty site could increase, resulting in a decrease in bone density ${ }^{19)}$.

There was no statistical difference in the operation time and blood loss between the AC and 3DC groups. In all patient groups, operation time and blood loss were required during adhesiolysis of the dura mater from the scalp. In the case of 3D printing flap cranioplasty, multiple holes can be tented with the screw fixation site, and the procedure required during operation has been shortened. In terms of patient satisfaction, all the patients were generally satisfied. In the case of a 3D printing flap, additional manufacturing is possible according to the patient. As it is manufactured based on muscle atrophy and according to the opposite side of the skull, it can be customized to a shape that suits the skull defect. However, in 3D bone flaps, a $1 \mathrm{~mm}$ thin-cut CT examination must be additionally performed for preoperative production, and it has the disadvantages of extra cost and radiation exposure risk.

In this study, we evaluated cranioplasty using a 3D printed bone flap and an autobone flap. The 3DC group had a high skull defect coverage rate and a low complication rate.

This was a retrospective medical record study with few cases. 
However, many artificial materials made by $3 \mathrm{D}$ printing techniques are currently used in surgery, and we expect this technique to develop rapidly. Therefore, future studies are essential to 3D-printing medical materials.

\section{CONCLUSION}

Cranioplasty using a customized artificial bone flap made by a $3 \mathrm{D}$ printing technique was effective for covering skull defects with lower complication rates than the autobone technique.

\section{NOTES}

\section{Conflict of interest}

No potential conflict of interest relevant to this article was reported.

\section{Supplementary Materials}

Further details on supplementary materials are presented online (available at https://doi.org/10.32587/jnic.2021.00388).

\section{REFERENCES}

1. Honeybul S, Morrison DA, Ho KM, Lind CR, Geelhoed E. A randomized controlled trial comparing autologous cranioplasty with custom-made titanium cranioplasty. J Neurosurg 2017; 126:81-90.

2. Mukherjee S, Thakur B, Haq I, Hettige S, Martin AJ. Complications of titanium cranioplasty--a retrospective analysis of 174 patients. Acta Neurochir (Wien) 2014;156:989-998.

3. Dujovny M, Fernandez P, Alperin N, Betz W, Misra M, Mafee M. Post-cranioplasty cerebrospinal fluid hydrodynamic changes: magnetic resonance imaging quantitative analysis. Neurol Res 1997;19:311-316.

4. Chang V, Hartzfeld P, Langlois M, Mahmood A, Seyfried D. Outcomes of cranial repair after craniectomy. J Neurosurg 2010;112:1120-1124.

5. Kim JH, Hwang SY, Kwon TH, Chong K, Yoon WK, Kim JH. Defining "early" cranioplasty to achieve lower complication rates of bone flap failure: resorption and infection. Acta Neurochir (Wien) 2019;161:25-31.

6. Rosenthal G, Ivan Ng, Moscovici S, Lee KK, Lay T, Martin C. Polyetheretherketone implants for the repair of large cranial defects: a 3-center experience. Neurosurgery 2014;75:523-529.

7. Harris DA, Fong AJ, Buchanan EP, Monson L, Khechoyan D, Lam S. History of synthetic materials in alloplastic cranioplasty.
Neurosurg Focus 2014;36:E20.

8. 1li S, Pollastri G, Torcia E, Ferrari F, Albanese M, et al. Cranioplasty using acrylic material: a new technical procedure. J Craniomaxillofac Surg 2004;32:5-9.

9. Gilardino MS, Cabiling DS, Bartlett SP. Long-term follow-up experience with carbonated calcium phosphate cement (Norian) for cranioplasty in children and adults. Plast Reconstr Surg 2009;123:983-994.

10. Goh RC, Chang CN, Lin CL, Lo LJ. Customised fabricated implants after previous failed cranioplasty.J Plast Reconstr Aesthet Surg 2010;63:1479-1484.

11. Ghanaati S, Al-Maawi S, Conrad T, Lorenz J, Rössler R, Sader R. Biomaterial-based bone regeneration and soft tissue management of the individualized 3D-titanium mesh: An alternative concept to autologous transplantation and flap mobilization. J Craniomaxillofac Surg 2019;47:1633-1644.

12. Schmidt M, Holzbauer M, Kwasny O, Huemer GM, Froschauer S. 3D Printing for scaphoid-reconstruction with medial femoral condyle flap. Injury 2020;51:2900-2903.

13. Bonda DJ, Manjila S, Selman WR, Dean D. The recent revolution in the design and manufacture of cranial implants: modern advancements and future directions. Neurosurgery 2015;77: 814-824.

14. De La Peña A, De La Peña-Brambila J, Pérez-De La Torre J, Ochoa M, Gallardo GJ. Low-cost customized cranioplasty using a 3D digital printing model: a case report. 3D Print Med 2018;4:4.

15. Morales-Gómez JA, Garcia-Estrada E, Leos-Bortoni JE, Delgado-Brito M, Flores-Huerta LE, De La Cruz-Arriaga AA, et al. Cranioplasty with a low-cost customized polymethylmethacrylate implant using a desktop 3D printer. J Neurosurg 2019;130: 1721-1727.

16. Ehrlich G, Kindling S, Wenz H, Hänggi D, Schulte DM, Schmiedek $\mathrm{P}$ et al. Immediate titanium mesh implantation for patients with postcraniotomy neurosurgical site infections: safe and aesthetic alternative procedure? World Neurosurg 2017;99:491499.

17. Kessler LA, Novelli PM, Reigel DH. Surgical treatment of benign intracranial hypertension--subtemporal decompression revisited. Surg Neurol 1998;50:73.

18. de França SA, Nepomuceno TB, Paiva WS, Andrade AF, Teixeira MJ, Tavares WM. Cranial autologous bone flap resorption after a cranioplasty: A case report. Surg Neurol Int 2018;9:61.

19. Lee SH, Yoo CJ, Lee UH, Park CW, Lee SG. Resorption of autogenous bone graft in cranioplasty: resorption and reintegration failure. Korean J Neurotrauma 2014;10:10-14. 